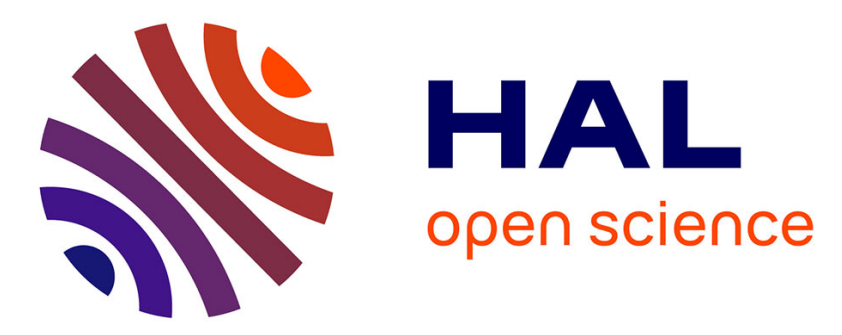

\title{
A new Voltage Measurement Probe for investigating Radiated Immunity Test
}

\author{
Alexandre Boyer, Sonia Ben Dhia, André Durier
}

\section{To cite this version:}

Alexandre Boyer, Sonia Ben Dhia, André Durier. A new Voltage Measurement Probe for investigating Radiated Immunity Test. EMC Europe 2020, Sep 2020, Rome (virtual), Italy. hal-02951842

\author{
HAL Id: hal-02951842 \\ https://hal.laas.fr/hal-02951842
}

Submitted on 29 Sep 2020

HAL is a multi-disciplinary open access archive for the deposit and dissemination of scientific research documents, whether they are published or not. The documents may come from teaching and research institutions in France or abroad, or from public or private research centers.
L'archive ouverte pluridisciplinaire HAL, est destinée au dépôt et à la diffusion de documents scientifiques de niveau recherche, publiés ou non, émanant des établissements d'enseignement et de recherche français ou étrangers, des laboratoires publics ou privés. 


\section{A new Voltage Measurement Probe for investigating Radiated Immunity Test}

\author{
A. Boyer, S. Ben Dhia \\ $L A A S-C N R S$ \\ Univ. de Toulouse, INSA, LAAS \\ Toulouse, France \\ alexandre.boyer@laas.fr, sonia.ben.dhia@laas.fr
}

\author{
A. Durier \\ IRT Saint-Exupéry \\ Toulouse, France \\ andre.durier@irt-saintexupery.com
}

\begin{abstract}
Diagnosis of failures induced during radiated immunity tests in anechoic chamber and validation of field-toline coupling models require measurements of the induced voltages at the input of electronic equipments. Although such measurements seem obvious at first sight, they can become complicated and erroneous due to measurement conditions. This paper describes the design a miniature high-frequency voltage probe to solve this issue. This probe is validated experimentally by a comparison with simulation.
\end{abstract}

Keywords-Radiated immunity, voltage probe, anechoic chamber, validation

\section{INTRODUCTION}

Radiated immunity (RI) tests in (semi)-anechoic chamber, as those defined by standards IEC61000-4-3, ISO11452-2 or DO160 are necessary to guarantee the safe operation of electronic equipments illuminated by electromagnetic disturbance. These tests require a precise control of all measurement parameters to ensure a sufficient repeatability of the results. Moreover, anticipating risks of failures needs an analysis of the field to line or equipment coupling mechanisms, making modeling and simulation unavoidable [1] [2] [3]. However, reliable measurement tools are also required to validate simulation results.

Field-to-line coupling models have been developed these last decades, such as [4] or [5]. They were usually validated by measurements based on TEM cell or capacitive coupling clamp, but hardly on anechoic chamber measurements. When it happened, it was current measurement with inductive coupling clamp and not voltages induced on line terminals or equipment input. Several practical difficulties arise when such a voltage measurement is done:

- the frequency range of these measurements (up to 1 or $6 \mathrm{GHz}$ ), imposing constraints on the measurement receiver.

- the presence of the measurement receiver may alter electromagnetic field locally. It may be placed outside the shielded room and connected to the device under test through coaxial cables

- the presence of cables contributes to a significant parasitic coupling of the incoming radiated disturbance, which compromises the repeatability of the RI test

- the measurement receiver must be as compact as possible and embed its own energy source to limit direct coupling of the incoming radiated disturbance.

The analysis of existing and usual measurement equipments used in EMC laboratory shows that no commercial solution exists to cover this need, certainly because it is not associated to any EMC compliance requirement. This paper aims at presenting a high frequency voltage measurement probe, miniature enough to limit parasitic coupling of the incoming disturbance, batterypowered, and designed only with off-the-shelf components. After a description of its principle and a presentation of its characteristics, an experimental validation is provided. The relevance of the measurement results of the voltage induced on a line under test during RI test is evaluated by a comparison with simulation.

\section{PRESENTATION AND CHARACTERIZATION OF THE VOLTAGE PROBE}

\section{A. Description of the design}

The first main constraint is the limitation of radiated disturbance coupling on the probe, which prohibits the use of coaxial cable and requires electro-optical conversion. However, the second main constraint is the frequency range, which must be at least $1 \mathrm{GHz}$ to cover usual RI test range. It reduces the number of affordable measurement devices. As only the amplitude of sine waveform is under concern, the easiest solution consists in using a RF power detector, which converts the amplitude of a RF signal into a continuous voltage. Commercial miniature RF detection module exists, such as Mini Circuit ZX 47-60-LN-S), but they are not battery powered and they have no optical output. Battery and output cables may contribute to radiated disturbance coupling. Thus, a home-made solution based on off-the-shelf components seems to be the only available solution at an acceptable cost.

Numerous commercial RF detector integrated circuits are available, with typical sensitivity ranging between -60 and $+20 \mathrm{dBm}$. On a $50 \Omega$ input, the voltage ranges between 220 $\mu \mathrm{V}$ and $700 \mathrm{mV}$. Their typical bandwidth starts at some $\mathrm{MHz}$ and stops at several $\mathrm{GHz}$ (sometimes at more than $10 \mathrm{GHz}$ ). These characteristics are compatible with requirements of RI tests. Moreover, these circuits are low-cost, low power, have a small temperature drift and are mounted in miniature packages.

TABLE I. MAIN CHARACTERISTICS OF RF DETECTOR LT5534 [6]

\begin{tabular}{|c|l|}
\hline Bandwidth & $50-3000 \mathrm{MHz}$ \\
\hline Dynamic range & $-60-0 \mathrm{dBm}$ \\
\hline Typical sensitivity & $41 \mathrm{mV} / \mathrm{dB}$ \\
\hline Temperature drift & $0.025 \mathrm{~dB} /{ }^{\circ} \mathrm{c}$ \\
\hline Power supply voltage & $2.7-5.25 \mathrm{~V}$ \\
\hline Current consumption & $7 \mathrm{~mA}$ \\
\hline Package & $6 \mathrm{pin} \mathrm{SC} 70$ \\
\hline
\end{tabular}


The RF detector LT5534 developed by Analog Devices [6] has been selected because of its small size and its simplicity. Its characteristics are given in Table I. This logarithmic-based detector has a bandwidth and a $60 \mathrm{~dB}$ dynamic range compatible with $\mathrm{RI}$ requirements.

The DC voltage provided by the RF detector has to be digitalized before its optical transmission. This task is ensured by the optical probe Langer A-100. With an input voltage range $0-10 \mathrm{~V}$ and a $25 \mathrm{kHz}$ bandwidth, it is compatible with the signal provided by the RF detector. The choice of the optical probe is justified by its compactness, its high resolution (12 bits), its low consumption ( $3 \mathrm{~mA})$ and its high robustness to electromagnetic disturbance $(>200 \mathrm{~V} / \mathrm{m})$. The detector also needs a input impedance matching network with a RC filter to stabilize and filter the output signal.

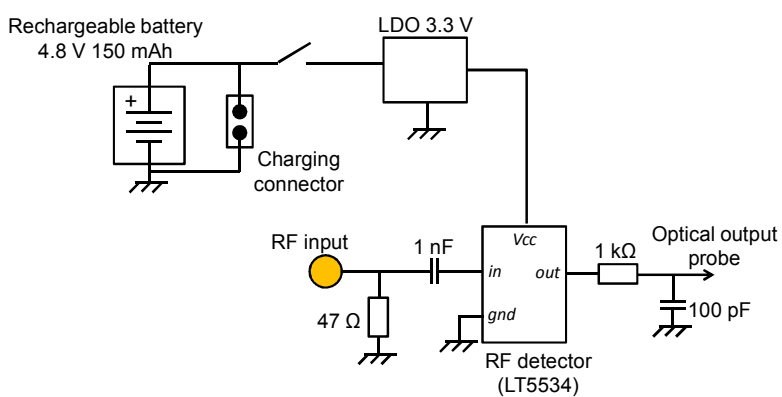

Fig. 1. Electrical schematic of the proposed voltage probe

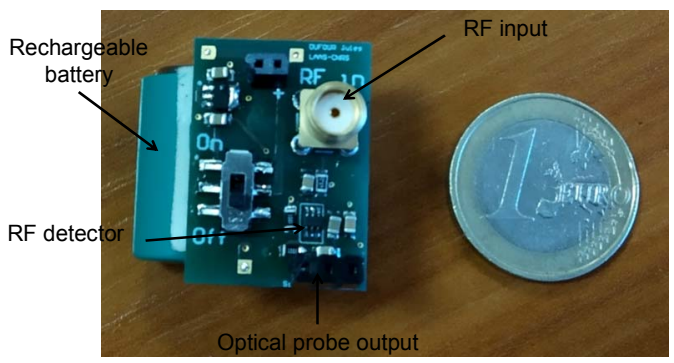

Fig. 2. Picture of the proposed voltage probe

The probe is powered by a $4.8 \mathrm{~V}$ miniature rechargeable microbattery, with a capacity of $150 \mathrm{~mA}$.h. The autonomy of the probe is estimated to 10 hours. The battery is followed by a low dropout regulator to provide a fixed $3.3 \mathrm{~V}$ voltage to the RF power detector and optical probe. The electrical schematic is presented in Fig. 1. A prototype has been developed on a 40 x $20 \mathrm{~mm}$ four-layer FR4 board (Fig. 2).

Preliminary illumination experience on the RF detector showed that the RF detector is sensitive to direct coupling of the incoming radiation, which may corrupt the measurement result. In order to preserve the integrity of the measurement results, the RF detector must be shielded. The sensitive active circuits, i.e. the RF detector and the LDO regulator, are placed within a shielded cabinet (Wurth WE-SHC) in order to minimize the direct coupling of the incident radiation on the voltage probe. The shielded cabinet is connected to an inner ground plane of the four-layer board. Except the RF input signal, all the signals which enter or leave the shielded cabinet are filtered by ferrite beads to limit the conducted coupling of the incident radiated disturbance to the $\mathrm{LDO}$ regulator and the $\mathrm{RF}$ detector.

\section{B. Characterization}

The sensitivity of the RF power detector varies according to the input signal power and frequency. A calibration is required prior to measurements. The characterization results of the probe sensitivity according to power and frequency are shown in Figs. 3 and 4. The calibration of the probe is based on the results of Fig. 3. The sensitivity is nearly constant up to $1 \mathrm{GHz}$ and loses $25 \%$ at $3 \mathrm{GHz}$. The probe can be used under the minimum frequency given by the detector manufacturer, at least at $10 \mathrm{MHz}$, as shown in Fig. 4. The probe input is $50 \Omega$ matched in order to limit return loss. The input reflection coefficient is shown in Fig. 5. It remains almost constant with a magnitude lower than $-25 \mathrm{~dB}$ up to $1.3 \mathrm{GHz}$. Above this frequency, it increases but remains less than $-8 \mathrm{~dB}$ at $3 \mathrm{GHz}$.

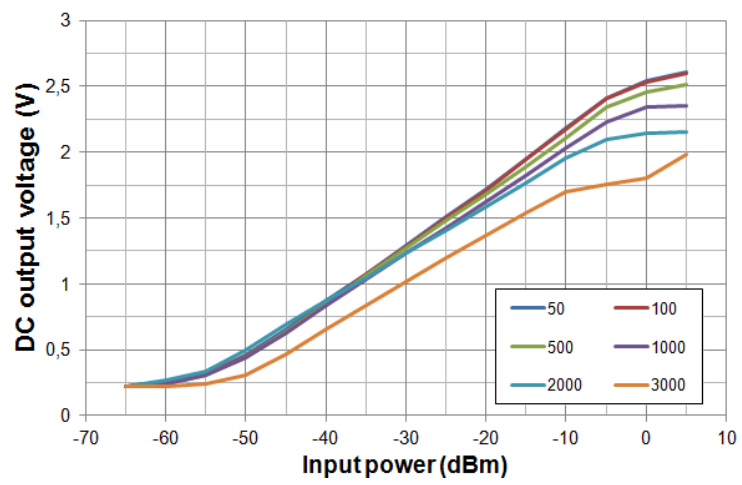

Fig. 3. Evolution of the probe output DC voltage according to the input signal power and frequency (in $\mathrm{MHz}$ )

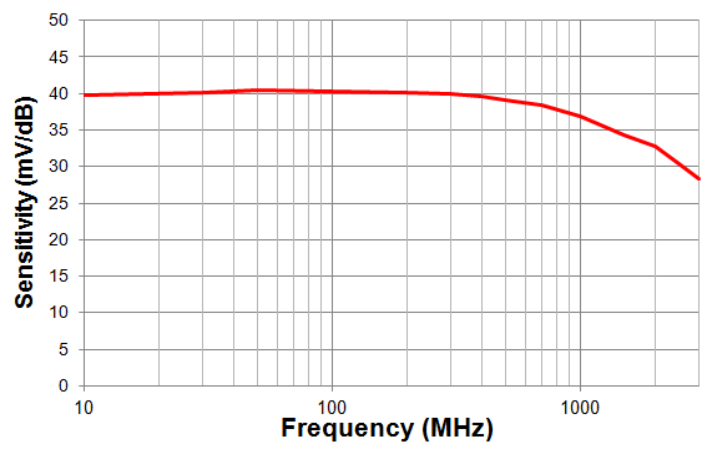

Fig. 4. Evolution of the pobe sensitivity vs. frequency

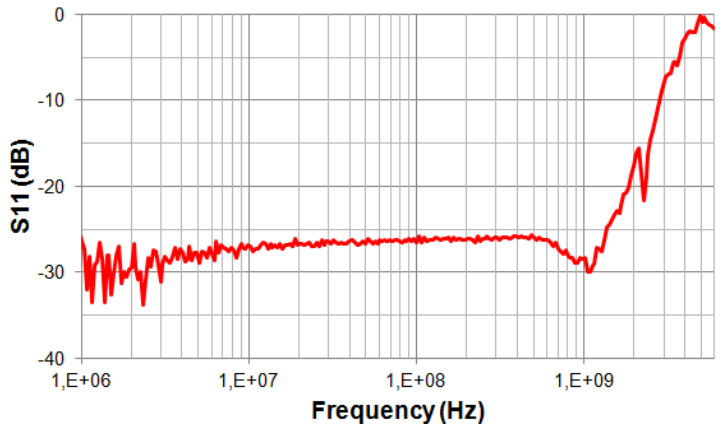

Fig. 5. Measurement of the reflection coefficient at the probe input

\section{COMPARISON OF MEASUREMENT METHODS}

\section{A. Case study description}

The relevance of voltage measurement provided by the proposed probe during RI test is tested. It consists in measuring the voltage induced at the terminal of a device 
under test (DUT) when it is illuminated by a radiated disturbance in semi-anechoic chamber. The measurement results with the proposed probe will not only be compared with simulation (see part IV), but also to direct measurement done with a spectrum analyzer connected to the DUT by a coaxial cable and placed outside the chamber. The DUT is a $75 \mathrm{~mm}$ long $2.5 \mathrm{~mm}$ wide $50 \Omega$ microstrip line designed on a 100x100 mm FR4 printed circuit board (PCB). The experimental set-up is described in Fig. 6.

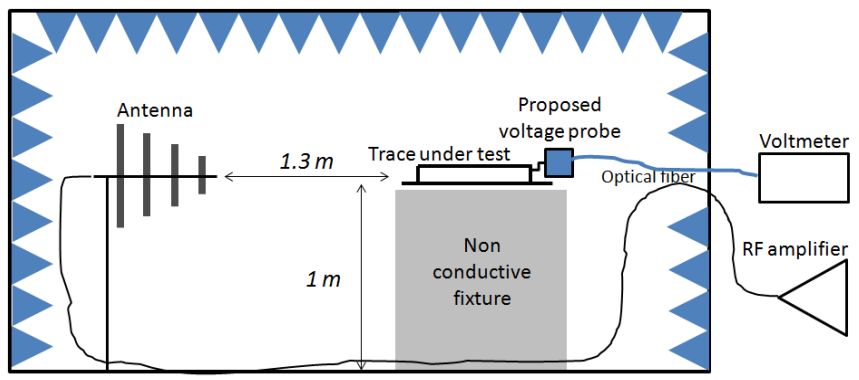

Fig. 6. Experimenal set-up: characterization of the induced voltage across the terminal of a line under test during radiated immunity test

The trace under test is placed on a non-conductive fixture at $1 \mathrm{~m}$ above the chamber ground. It is illuminated by a RF disturbance radiated by a log-periodic antenna placed at 1.3 $\mathrm{m}$. The antenna is excited by a sine waveform signal produced by a RF signal synthesizer and amplified by a 50 $\mathrm{W}$ power amplifier. The test frequencies cover the range 30 $1000 \mathrm{MHz}$. Tests have been done with vertically polarized electric field, whose magnitude is about $20 \mathrm{~V} / \mathrm{m}$. The E-field intensity is controlled by an electric field probe (model EP600 from Narda). Preliminary tests have proved that the field around the line under test could be considered as homogeneous. The uncertainty on the incoming field intensity is about $10 \%$, except between 80 and $200 \mathrm{MHz}$ where it reaches $25 \%$ due to chamber resonances.

\section{B. Characterization of the sensitivity of the probe to incoming radiated disturbance}

Initially, the voltage probe is placed in the anechoic chamber and is not connected to any trace under test. This measurement aims at characterizing the error introduced by a direct coupling of the incoming radiation on the probe, which may disturb its operation. The probe is placed at $1.3 \mathrm{~m}$ to the injection antenna. It is placed horizontally; the RF connector is oriented to the antenna. The power injected to the antenna is set to produce a constant electric field. The incoming wave is vertically polarized. Fig. 7 shows the evolution of the power measured by the probe in this configuration.

The power measured by the probe is not affected by the incoming radiation up to $1.2 \mathrm{GHz}$. The measured power is less than $-65 \mathrm{dBm}$, i.e. the sensitivity threshold of the RF detector. Above $1.2 \mathrm{GHz}$, the effect of a direct coupling to the probe becomes visible. A peak appears at $1.7 \mathrm{GHz}$, where the measured power is equal to $-39 \mathrm{dBm}$, i.e. a voltage of $2.5 \mathrm{mV}$ on a $50 \Omega$ load. This power remains small in comparison to the voltage that may be induced by radiated coupling on large structures. This peak is certainly due to the resonance of the cavity formed by the inner ground plane of the PCB and the shielded cabinet placed around the RF detector and the LDO regulator. The input-output filtering and the shielding of the probe PCB should be improved to reduce the sensitivity of the probe, especially the RF detector, to the direct coupling of radiated disturbance.

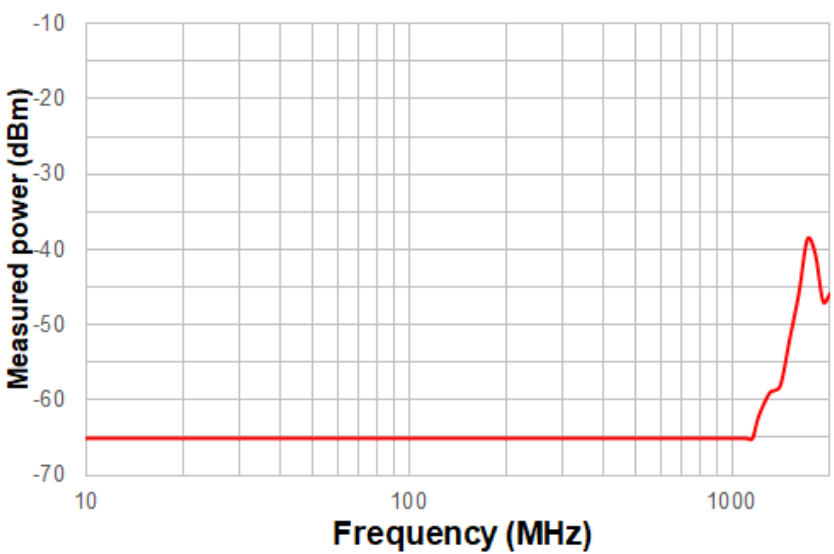

Fig. 7. Characterization of the sensitivity of the probe to the incoming radiated disturbance

\section{Measurement results and analysis}

Measurements have been done for two orientations of the line under test: the PCB is placed horizontally and the illuminated trace is oriented perpendicularly to the antenna. Three measurements have been performed and compared: with the proposed voltage probe (after calibration) and two others with a spectrum analyzer and two types of $2 \mathrm{~m}$ long $50 \Omega$ coaxial cables. They differ from their shielding: the first cable has only one shielding layer while the second has two shielding layers. The position of both cables in the chamber is nearly identical. Measurement results are presented in Fig. 8.

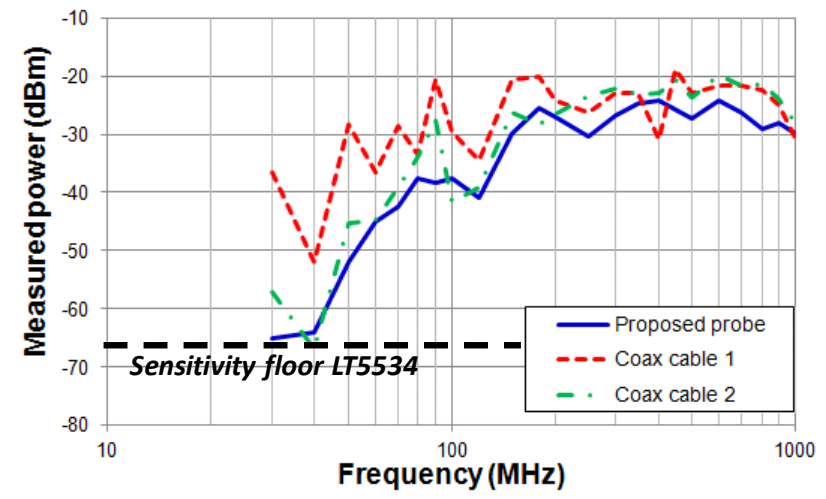

Fig. 8. Comparison of the power coupled on the line under test measured with the three different measurement methods

Although the measurement types present similar trends and orders, significant differences are visible, especially under $200 \mathrm{MHz}$. In this frequency range, the far-field hypothesis is not valid and the chamber resonates. These differences are due to the coaxial cables that contribute to radiated disturbance coupling as parasitic antenna. The coupled field is not only related to the characteristics of the trace under test, but also to those of the coaxial cables which connects the trace under test to the spectrum analyzer.

The observed frequency-dependent variations with both measurements made with spectrum analyzer are explained by the length of cables which introduces multiple resonances. Measurements made with our miniature probe present less variations because of its small size and its low contribution to incoming field coupling. The measured variations are due to non-constant field over the frequency range. 
It must be underlined that the measurements done with both types of cable show differences that can reach 10 to 20 $\mathrm{dB}$, especially below $200 \mathrm{MHz}$. Field coupling depends on the common-mode impedance of cables which is related to the nature of the shielding. Any change in the internal construction of the cable and its position induces a large measurement variability, unlike the proposed measurement probe.

\section{SIMULATION AND VALIDATION}

Experimental results show that the proposed probe improves the repeatability of the measurement of the coupled voltage on an illuminated line. However, the validity of the measured voltage must be tested, so that it is compared to prediction based on a far-field coupling model.

\section{A. Description of the model}

The trace under test was initially characterized with a vector network analyzer to extract its per-unit-length (pul) parameters. These values were also compared with theoretical pul parameters, computed with the freeware ICEMC [7], which provides a good agreement. Then a Taylor model was built to determine the voltage coupled on its terminals when it is illuminated [4]. The field recorded by the electric-field probe is used as excitation values in the simulation. Fig. 9 illustrates the geometrical and the electrical equivalent models. Vertical electric $E_{Z}$ and tangential magnetic $\mathrm{H}_{\mathrm{x}}$ fields contribute to generate distributed electromotive force and current along the line, as given by (1) and (2). They contribute to the voltages induced across each terminal of the line, whose expressions are given by (3) and (4).

$$
\begin{gathered}
d V_{H}=j \omega \mu_{0} h H_{X} d y \\
d I_{E}=j \omega h c E_{Z} d y \\
V_{1}(\omega)=\int_{0}^{L} \frac{\left(1+\Gamma_{1}\right) e^{-k y}}{1+\Gamma_{1} e^{-2 k y}} \frac{Z_{e q 1}}{Z_{e q 1}+Z_{e q 2}}\left(d V_{H}+Z_{e q 2} d I_{E}\right) d y \\
V_{2}(\omega)=\int_{0}^{L} \frac{\left(1+\Gamma_{2}\right) e^{-k(L-y)}}{1+\Gamma_{2} e^{-2 k(L-y)}} \frac{Z_{e q 2}}{Z_{e q 1}+Z_{e q 2}}\left(d V_{H}-Z_{e q 1} d I_{E}\right) d y
\end{gathered}
$$

where $Z_{\text {eq1 }}$ and $Z_{\text {eq2 }}$ are the terminal load impedances seen from a point $y$ of the line ((5) and (6)). $\Gamma_{1}$ and $\Gamma_{2}$ are the reflection coefficients of termination load $Z_{\mathrm{L} 1}$ and $Z_{\mathrm{L} 2}, \mathrm{k}$ is the wavenumber and $Z_{c}$ the characteristic impedance of the microstrip line.

$$
\begin{gathered}
Z_{e q 1}(y)=Z_{C} \frac{Z_{L 1}+Z_{C} \operatorname{th}(k y)}{Z_{C}+Z_{L 1} \operatorname{th}(k y)} \\
Z_{e q 2}(y)=Z_{C} \frac{Z_{L 2}+Z_{C} \operatorname{th}(k(L-y))}{Z_{C}+Z_{L 2} \operatorname{th}(k(L-y))}
\end{gathered}
$$

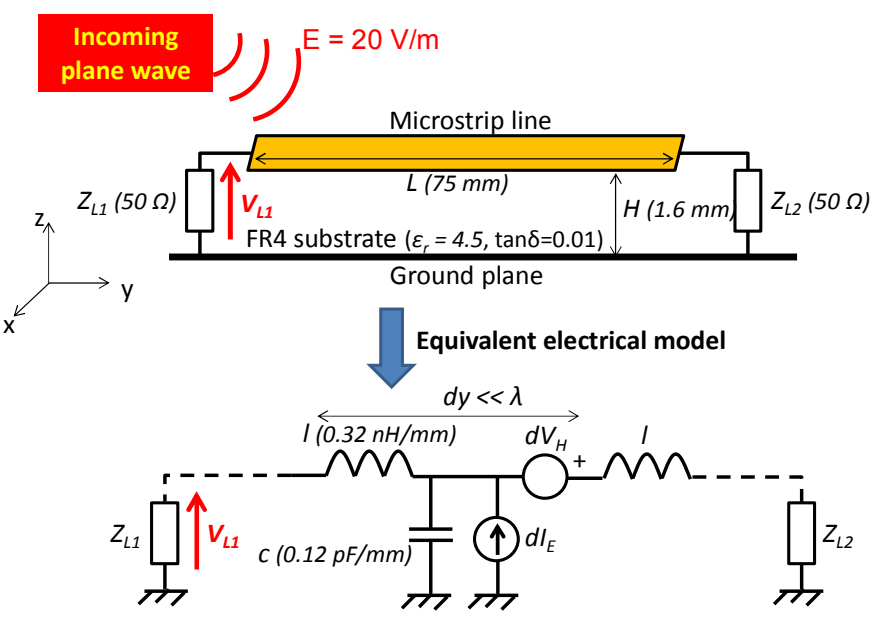

Fig. 9. Equivalent model of the illuminated trace (Taylor model)

However, Taylor model is not sufficient to take into account the presence of the ground plane and the PCB substrate. They modify the distribution of the $\mathrm{E}$ and $\mathrm{H}$ fields around the line, due to the superposition of the incoming excitation wave and the reflected waves at the substrate-air interface and ground plane. A correction is provided to compute the distribution of $\mathrm{E}$ and $\mathrm{H}$ fields within the substrate, based on the model described in [8]. The considered geometrical model is described in Fig. 10. The substrate is illuminated by an incident wave, with an electric field value $E_{\text {inc }}$ and an angle of arrival $\theta_{2}$. Here, the model is only described when the $\mathrm{E}$ field is in the $\mathrm{xz}$ plane, but equivalent formulations can be found when the $\mathrm{E}$ field is perpendicular to the xy plane. Within the substrate (medium $1)$, the $\mathrm{E}$ and $\mathrm{H}$ field complex amplitudes are given by (7) to (9).

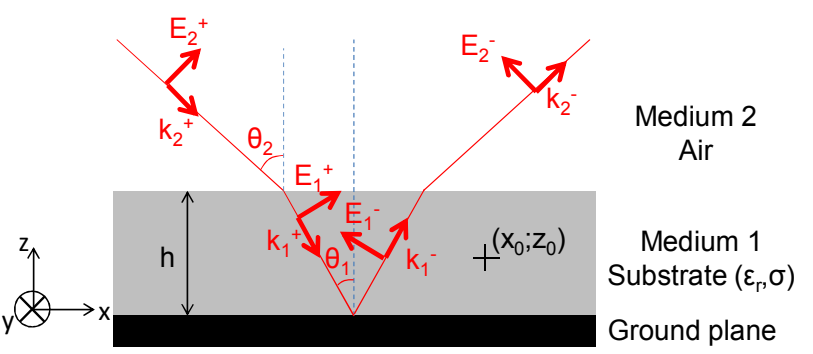

Fig. 10. Incident and reflected waves on a two-layer planar structure

$$
\begin{gathered}
E_{1 x}=C_{1}^{+}\left(e^{j k_{1} z_{0} \cos \theta_{1}}-e^{-j k_{1} z_{0} \cos \theta_{1}}\right) e^{-j k_{1} x_{0} \sin \theta_{1}} \\
E_{1 z}=C_{1}^{+} \tan \theta_{1}\left(e^{j k_{1} z_{0} \cos \theta_{1}}+e^{-j k_{1} z_{0} \cos \theta_{1}}\right) e^{-j k_{1} x_{0} \sin \theta_{1}} \\
H_{1 y}=\frac{-k_{1} C_{1}^{+}}{\omega \mu_{0} \cos \theta_{1}}\left(e^{j k_{1} z_{0} \cos \theta_{1}}+e^{-j k_{1} z_{0} \cos \theta_{1}}\right) e^{-j k_{1} x_{0} \sin \theta_{1}}
\end{gathered}
$$

$\mathrm{C}_{1}{ }^{+}$is the complex amplitude of the incident wave in the substrate and is given by (10), where $\mathrm{k}_{1}$ and $\mathrm{k}_{2}$ are the wavenumbers in media 1 and 2 ((12) and (13)). 


$$
\begin{gathered}
C_{1}^{+}=E_{i n c} \frac{2 \cos \theta_{2} \sin 2 \theta_{1} \cdot e^{j k_{2} h \cos \theta_{2}}}{D} \\
D=\left(\sin 2 \theta_{2}+\sin 2 \theta_{1}\right) e^{j k_{1} h \cos \theta_{1}}+\left(\sin 2 \theta_{2}-\sin 2 \theta_{1}\right) e^{-j k_{1} h \cos \theta_{1}} \\
k_{1}=\omega \sqrt{\mu_{0} \varepsilon_{0}\left(\varepsilon_{r}-j \frac{\sigma}{\omega \varepsilon_{0}}\right)} \\
k_{2}=\omega \sqrt{\mu_{0} \varepsilon_{0}}
\end{gathered}
$$

The model has been implemented in the freeware ICEMC [7].

\section{B. Comparison measurement - simulation}

A comparison between measurements made with the proposed probe and simulations is shown in Fig. 11, for two orientations of the trace under test. An acceptable agreement is found between measurement and simulation. The maximum gap is observed between 100 and $200 \mathrm{MHz}$. In this frequency range, the far-field condition is not met perfectly and chamber resonance enhances measurement variability. This result confirms the relevance of the measurement done with our proposed probe for the characterization of the voltage induced on a line illuminated in a (semi-)anechoic chamber.

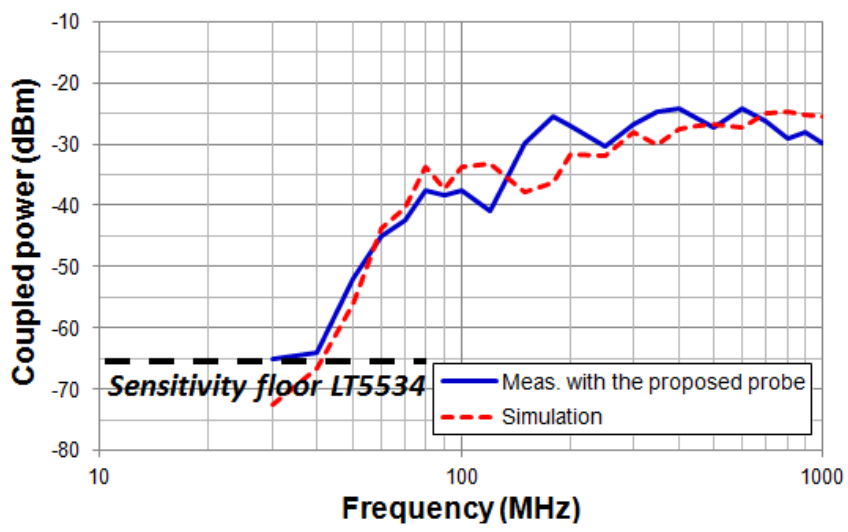

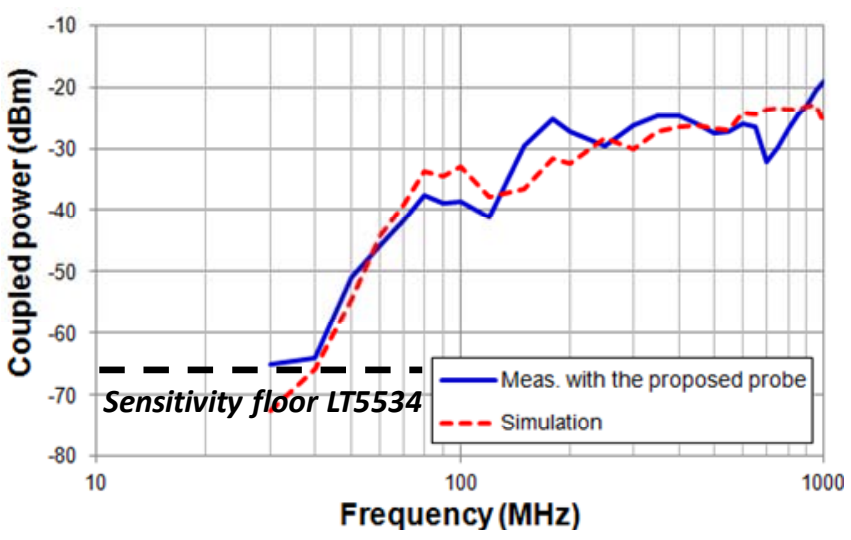

Fig. 11. Comparison between measurement and simulation of the voltage coupled on the trace under test: orientation 1 (top), orientation 2 (bottom)

\section{CONCLUSION}

This paper has presented a high frequency voltage measurement probe dedicated to the validation of field-toline coupling models during radiated immunity tests in anechoic chamber. The probe is made only with off-the-shelf components. It is battery-powered and miniature enough to limit the direct coupling of incoming fields. Comparisons with other measurement methods and simulations have proven the validity of this measurement device.

\section{REFERENCES}

[1] A. Durier, S. Ben Dhia, T. Dubois, "Comparison of voltages induced in an electronic equipment during far field and near field normative radiated immunity tests", EMC Europe 2019, Barcelona, Spain, Sept 2019.

[2] S. Caniggia, F. Maradei, "SPICE-like models for the analysis of the conducted and radiated immunity of shielded cables", IEEE Trans. on EMC, vol. 46, no. 4, pp. 606-616, Nov. 2004.

[3] M. Leone, H. L. Singer, "On the coupling of an external electromagnetic field to a printed circuit board trace", IEEE Trans. on EMC, vol. 41, no. 4, pp. 418-424, Nov 1999

[4] C. D. Taylor, R. S. Satterwhite, W. J. Harrison, "The response of terminated two-wire transmission line excited by a non uniform electromagnetic field", IEEE Trans. on Antennas and Propagation, vol. AP-13, pp. 987-989, 1965.

[5] C. R. Paul, "Frequency response of multiconductor transmission lines illuminated by an electromagnetic field", IEEE Trans. on EMC, vol. EMC-18, no. 4, pp. 183-190, 1976.

[6] LT5534 - 50MHz to $3 \mathrm{GHz}$ RF Power Detector with 60dB Dynamic Range, Datasheet, Linear Technology, 2004.

[7] IC-EMC v2.9, freeware available on www.ic-emc.org.

[8] K. J. Scott, "Practical Simulation in Printed Circuit Boards and Related Structures - First Edition", Research Studies Press, 1994. 